\title{
Traduire
}

Ine autre perspective sur $r$ tadadciction

Revue française de la traduction

$240 \mid 2019$

Quand la politique s'en mêle

\section{Les pièges du langage totalitaire : traduire le nazisme}

Petit manuel de survie

\section{Olivier Mannoni}

\section{(2) OpenEdition \\ Journals}

\section{Édition électronique}

URL : http://journals.openedition.org/traduire/1654

DOI : $10.4000 /$ traduire.1654

ISSN : 2272-9992

\section{Éditeur}

Société française des traducteurs

Édition imprimée

Date de publication : 20 juin 2019

Pagination : 36-44

ISSN : 0395-773X

\section{Référence électronique}

Olivier Mannoni, «Les pièges du langage totalitaire : traduire le nazisme », Traduire [En ligne], 240 | 2019, mis en ligne le 20 juin 2019, consulté le 07 février 2020. URL : http://journals.openedition.org/ traduire/1654; DOI : 10.4000/traduire.1654 


\section{Les pièges du langage totalitaire: traduire le nazisme}

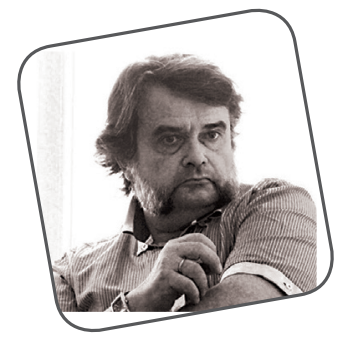

Petit manuel de survie

\section{Olivier Mannoni}

\section{Mauvaises rencontres}

Mon premier véritable contact de traducteur avec la langue du nazisme s'est fait par le biais de l'éthologie. L'excellent éditeur Abel Gershenfeld, à l'époque aux éditions Odile Jacob, m'avait demandé de lire pour lui le manuscrit encore inachevé d'un ouvrage sur les babouins hamadryas'. Heureux du résultat et ne reculant pas devant les coq-à-l'âne vertigineux, Abel m'informa qu'en l'attente de la fin du manuscrit, il souhaitait me confier la traduction d'un livre portant sur d'autres animaux tout aussi agressifs, mais nettement moins sociaux... les médecins et scientifiques nazis². Étonné, mais heureux d'entamer cette première collaboration avec la prestigieuse maison d'édition, je plongeai donc dans ce travail avec une certaine inquiétude.

On connaît l'histoire de la médecine et de la science nazies, de leurs savants fous, de leurs expériences démentes sur des détenus et des enfants. Mais ce qui m'a marqué n'est pas tant l'atrocité 
des actes commis, tels qu'ils sont racontés dans la première partie de cet ouvrage, que ce qu'on peut lire dans la deuxième: les entretiens réalisés par l'auteur avec une dizaine d'anciens assistants des médecins ou scientifiques de Hitler, assistants devenus des professeurs et chercheurs renommés au moment où Benno Müller-Hill avait mené son enquête. Ce qui était ahurissant, c'était la facilité avec laquelle ces hommes, tout en sachant combien de souffrance, d'épanchement de sang et d'atrocités leurs patrons de l'époque - et eux-mêmes - avaient provoqués, justifiaient leurs actes: tout avait eu lieu, disaient-ils, dans le respect de l'esprit scientifique, et pour le bien de l'humanité. Je découvris alors de quelle gangue verbale le nazisme avait réussi à s'entourer pour présenter, dissimuler ou exalter sa propre réalité.

Les racines de la langue nazie remontent loin dans l'histoire, bien avant la naissance du Parti national-socialiste allemand des ouvriers, le NSDAP. Et même bien avant les premiers pas politiques de l'ancien première classe Adolf Hitler et de sa petite clique. Elle s'enracine dans un mouvement très large auquel on a donné le nom intraduisible de völkisch, mélange de nationalisme ethnique, de retour au terroir, d'ésotérisme, d'antisémitisme, de paganisme et de quelques autres ingrédients. La littérature indigeste de ce mouvement informe a semé quelques graines qui ont germé par la suite: un certain goût pour l'emphase, pour la vibration patriotique, l'apologie de la violence et l'obscurité du langage. Tous ces éléments ont été repris ultérieurement par les auteurs de la "révolution conservatrice». Niekisch, Freyer, Spengler ou Moeller van den Bruck en ont fait un abondant usage, tout comme un certain Ernst Jünger, qui donne par exemple dans un texte boursouflé de 1930 toute la mesure de son antisémitisme ${ }^{3}$. On retrouvera pendant toute l'histoire du nazisme ce singulier mélange de flou ésotérique, de délires païens, de raisonnements brutaux, pour ne pas dire brutalistes, portés par un ton exalté et des élans martiaux. Cela s'exprime, bien entendu, de la manière la plus claire dans Mein Kampf, ouvrage que Hitler écrit au milieu des années 1920 dans la forteresse de Landsberg, où une tentative de putsch, en 1923, lui a

3. "La connaissance et la réalisation de la forme allemande singulière écartent d'elle la forme du Juif. [...] Mais dans la mesure même où la volonté allemande prend forme et devient plus vive, même la plus faible des folies, vouloir être Allemand en Allemagne, deviendra pour un Juif un vœu toujours plus idéaliste, et il se trouvera face à sa dernière alternative: en Allemagne, soit être Juif, soit ne pas être.» Cité in Josef Brever, La Révolution conservatrice, trad. O. M., Paris, MSH, 1996. 
valu de faire un (assez bref et très confortable) séjour. La langue de Hitler est comme le mouvement d'une croix gammée: elle hypnotise et elle broie. Elle hypnotise parce que son fonctionnement circulaire assoupit en quelque sorte le cerveau, le fait s'enrouler sur lui-même comme la "pensée» qu'il a en face de lui jusqu'à ce qu'elle lui assène le coup idéologique final en deux ou trois phrases brèves et percutantes. Mein Kampf respecte les codes du style völkisch, obscur et ampoulé, et les met au service de démonstrations aussi soporifiques qu'efficaces.

La confusion et l'opacité ne sont cependant ni les seules caractéristiques ni les traits essentiels de la langue nazie. Celle-ci s'est adaptée aux quatre rôles principaux qu'elle devait remplir: séduire, terroriser, modifier le réel, dissimuler la réalité. Confronté à ce caméléon linguistique, le traducteur a fort à faire. Il devra constamment s'efforcer de ne pas se laisser emporter par l'emphase de son texte - par exemple à la fin du premier tome de Mein Kampf ou dans les envolées lyriques du Journal de Goebbels: ces deux grands manipulateurs s'entendaient comme peu de leurs contemporains à exciter l'imagination et l'enthousiasme de leurs lecteurs ou de leurs auditeurs pour mieux anesthésier leur raison et leur faculté de juger. Mais il devra aussi descendre dans les tréfonds de cette langue s'il ne veut pas en masquer les relents nauséabonds.

\section{Séduire et habituer}

Devant le traducteur des textes sur le nazisme, deux strates de langage se superposent: celle de l'étude historique, qui applique des mots et des définitions à des réalités; et celle du texte source, qui peut utiliser ces mêmes mots dans un sens qui ne correspond pas ou plus à ce vocabulaire académique. Le Vernichtung utilisé par Hitler dans Mein Kampf pour désigner l'«anéantissement» supposé de l'Allemagne par le Traité de Versailles n'a ainsi plus rien à voir avec le sens de ce terme lorsque Hitler nomme l'ennemi militaire à «détruire», ni quand on l'emploie dans l'expression Vernichtungslager, créée après la fin du nazisme pour désigner les «camps d'extermination». Le mot fanatisch que l'on retrouve avec une fréquence exceptionnelle chez Goebbels, Himmler ou Hitler revêt chez eux une notion positive, radicalement opposée à celle, négative, que les historiens utilisent pour qualifier l'état d'esprit des foules exaltées qui soutenaient le nazisme. 
II est d'autant plus important de tenir compte du rôle que l'on a voulu faire jouer, à l'époque, à ces mots du langage quotidien. Victor Klemperer a montré dans sa célèbre étude $L T I$, mais plus encore dans ses admirables journaux ${ }^{4}$, comment la "langue du Troisième Reich» s'est insinuée dans la vie quotidienne pour en contaminer et, finalement, en transformer la substance jusqu'à l'absurde: un mouvement des «déformateurs du langage» s'était même constitué au sein du nazisme, et s'était donné pour but de purifier l'allemand pour qu'il ne contienne plus que des racines germaniques. Goebbels mit un terme définitif à cette plaisanterie quand ils s'avisèrent de changer le mot Nase, le «nez», jugé trop latin, par le très improbable, mais germanique, Gesichtsturm, «la tour du visage».

Mais la manipulation du langage n'eut pas que des aspects grotesques. Comme de l'huile sur du papier absorbant, les concepts centraux du nazisme se répandirent partout. Le très ambigu mot Volk, qui désigne comme en français aussi bien le «peuple» au sens social que "l'ethnie» au sens racial, fut ainsi mis à toutes les sauces: Volksgemeinschaft ("communauté du peuple»), Volksgenosse ("camarade du peuple»). Mais si ces termes-là étaient destinés à constituer une communauté exclusive dont ne faisaient partie ni les membres de «races inférieures» ni les personnes ne partageant pas l'amour du nazisme, d'autres étaient là pour enraciner cette communauté dans la vie quotidienne et l'esprit des Allemands: la Volkswagen était ainsi la "voiture du peuple» et le Volksempfänger le «récepteur radio du peuple». Victor Klemperer a dressé dans LTI un inventaire précis de cette contamination du langage dont certains agents pathogènes sévissent de nouveau, aujourd'hui, dans l'ensemble de l'Europe.

\section{Terroriser}

Mais cette opération de séduction et d'imprégnation du langage n'était pas tout: le nazisme, dès le début, a aussi voulu terroriser ses adversaires. On connaît la célèbre scène du Dictateur

4. Victor Klemperer, LTI, la langue du $/ l^{\text {e }}$ Reich, traduit de l'allemand par Élisabeth Guillot, Paris, Albin Michel, 1996. Id., Mes soldats de papier et Je veux témoigner jusqu'au bout, traduit de l'allemand par Gislain Riccardi et al., Paris, Éditions du Sevil, 2000. Voir également le livre essentiel de Peter Reichel, La Fascination du nazisme, trad. O. M., Paris, Odile Jacob, 1993. 
où Chaplin est littéralement soufflé par les mots de Hitler, crachés dans la rue par un haut-parleur. De la part de Chaplin, qui a sans doute, à l'époque, réalisé la plus fine des études sur la nature profonde du nazisme, ce gag n'en était pas vraiment un. L'autre face de la langue nazie, à côté de la séduction et de la contamination, était l'usage de la dimension terrifiante que l'on peut donner à la langue allemande. II suffit pour cela de la rendre rugueuse, claquante, cinglante. C'est ce à quoi s'employa le «mouvement» nazi dès sa naissance, ou presque. Dans le choix, par exemple, des dénominations de ses unités de combat, la Sturmabteilung, la "SA», qui signifiait «section d'assaut», mais qui pouvait également se lire «section tempête» et claquait de toute façon comme un coup de fouet, à l'instar de la Schutzstaffel, la SS ("escadron de protection») dont les grades étaient un grotesque festival de chuintantes et d'allitérations aussi ronflantes qu'intraduisibles (SS-Obersturmbannführer, "chef de section d'assaut supérieur», SS-Rottenführer, littéralement "chef de horde», etc.). Cette violence quasi poétique, au sens premier du terme, se retrouvait dans tous les discours de propagande du mouvement, puis du régime. II s'agissait à la fois de terroriser l'adversaire ou l'ennemi et de rassembler ses propres "fanatiques» autour d'une force brute, d'une énergie destructrice. Une phrase de Goebbels, prononcée lors du fameux discours du Sportpalast sur la guerre totale le 18 février 1943, en constitue un parfait exemple, parmi d'autres, innombrables - on gagnera à la lire à voix haute:

Wir Deutschen sind gewappnet gegen Schwäche und Anfälligkeit, und Schläge und Unglückställe des Krieges verleihen uns nur zusätzliche Kraft, feste Entschlossenheit und eine seelische und kämpferische Aktivität, die bereit ist, alle Schwierigkeiten und Hindernisse mit revolutionärem Elan zu überwinden.

Outre le fait que la syntaxe est d'une lourdeur de Panzer, on note que cette phrase interminable multiplie les chuintantes (Schwäche, Schläge, Schwierigkeiten, Entschlossenheit) et les claquements de consonnes en «p» et en "k». II s'agit de donner un corps de guerrier à la langue pour qu'elle porte son message de guerre:

Nous, Allemands, sommes armés pour faire face à la faiblesse et à la vulnérabilité; les coups et les malchances de la guerre ne font 
que nous donner une force supplémentaire, une ferme détermination et une activité spirituelle et combative prêtes à surmonter toutes les difficultés et tous les obstacles avec un élan révolutionnaire ${ }^{5}$.

On distingue tout juste, et laborieusement, le sens de cette diatribe. Mais là n'était pas la question: elle était conçue pour faire à la fois vibrer et trembler les spectateurs du palais des Sports de Berlin.

\section{Dissimuler}

Conscients du caractère abominable et sans précédent du génocide qu'ils étaient en train de commettre, les nationauxsocialistes ont dès le début multiplié les mesures de précaution pour dissimuler d'abord leurs intentions, puis leurs actes. Les termes destinés à masquer la réalité du massacre étaient nombreux et furent rodés dès le début du régime, pour lequel l'éviction, pour le moins, des Juifs hors d'Allemagne, fut d'emblée un objectif et une obsession. Mais on cherche en vain dans les textes officiels ou de propagande des mots comme «déportation» ou «extermination». L'euphémisme "Solution finale» (Endlösung) utilisé après la conférence de Wannsee était l'un des derniers maillons d'une longue chaîne de dissimulations linguistiques qui jouaient sur l'ambivalence du vocabulaire. Vernichtung, nous l'avons vu plus haut, était ainsi employé alternativement pour désigner l'«anéantissement» économique de l'Allemagne (notamment dans Mein Kampf), la "destruction» des troupes ennemies, puis l'«extermination». Entfernen ("éloigner», comme on éloigne un objet sur une table), ausräumen ("évacuer»), Transport, Lager ("camp») étaient autant de termes destinés à masquer sous une ambiguïté volontaire la réalité du génocide et des déportations. La correspondance entre Himmler et sa femme, ou encore les entretiens délirants qu'Eichmann eut en Argentine avec une bande d'anciens et de nouveaux nazis ${ }^{6}$ en sont deux exemples frappants. Lors de ces discussions, Eichmann évoque avec clarté les déportations qu'il

5. Notre traduction. Le texte allemand de ce discours se trouve par exemple ici: Www.1000 dokumente.de/ 6. Katharina Himmler (éd.). Himmler d'après sa correspondance avec sa femme, trad. O. M., Paris, Plon, 2014; Bettina Stangneth, Eichmann avant Jérusalem, trad. O. M., Paris, Calmann-Lévy, 2017. 
qualifie de «particulièrement gentilles et coquettes ${ }^{7}$ », mais tout son discours, presque jusqu'à la fin, visera à masquer la dimension du massacre et son caractère même:

Je veux vous dire aujourd'hui que sur tout le parcours j'ai vu deux cadavres, c'étaient de vieux Juifs, c'est évident, on ne fait pas d'omelette sans casser des œufs. N'y en a-t-il pas eu, des œufs cassés, quand des contingents d'Allemands encore plus nombreux se sont mis en marche depuis l'Est après $45^{\circ}$ ?

Les «transports» désignant en réalité les déportations violentes apparaissent ainsi comme de simples déplacements de population. Et l'on pourrait bien sûr multiplier les exemples à l'infini. Notons également l'un des traits majeurs de la langue nazie: son incohérence, son absence d'épine dorsale syntaxique, qui permettent tous les glissements sémantiques et logiques ${ }^{9}$.

À la fin de ces entretiens, à la stupéfaction de ses interlocuteurs qui souhaitaient utiliser son témoignage pour propager la légende révisionniste selon laquelle il n'y aurait eu que 300000 morts dans les camps nazis, Eichmann finira par révéler que l'objectif n'était pas les six millions de Juifs, Tziganes, etc., assassinés dans les camps, mais... dix millions de morts. C'est I'un des rares textes où un haut responsable nazi (ce qu'était bien Eichmann, contrairement à ce qu'affirme la thèse du fonctionnaire invisible et discret) finit par mentionner clairement la nature réelle du génocide. À la fin de la guerre, dans deux terrifiants discours tenus devant les officiers SS à Posen (Poznan), Himmler s'était lui aussi adressé sans voile à ses hommes:

Je vous demande avec insistance d'écouter simplement ce que je dis ici en petit comité et de ne jamais en parler. La question suivante nous a été posée: "Que fait-on des femmes et des enfants? - Je me suis décidé et j'ai là aussi trouvé une solution évidente. Je ne me sentais en effet pas le droit d'exterminer (auszurotten) les hommes - c'est à dire, donc, de les tuer (umbringen) ou de les faire tuer - et de laisser grandir les enfants qui se vengeraient sur nos enfants et nos descendants. Il a fallu prendre la grave décision de faire disparaitre ce peuple de la terre. Pour l'organisation qui dut accomplir

7. «Entretiens Sassen», 68, 9, in B. Stangneth, op. cit.

8. Ibid., 23, 4 .

9. Incohérence qu'on retrouve aujourd'hui aussi bien dans les discours populistes européens que dans les diatribes et les tweets de Donald Trump aux États-Unis. 
cette tâche, ce fut la chose la plus dure que nous ayons eve jusqu'à présent. Cela a été accompli. [...] Nous aurons réglé la question juive dans les pays que nous occupons d'ici à la fin de l'année ${ }^{10}$.

\section{Traduire}

On l'aura compris, chacun de ces trois aspects force le traducteur à poser des questions nouvelles et à trouver des solutions différentes. Le principe de précaution est ici la règle absolve: chaque mot, chaque phrase, chaque "concept» doit être examiné non seulement quant à son sens, mais à l'aune de son histoire, de son contexte politique ou militaire, de sa place dans la chaîne de dissimulation et de son objectif dans la propagande. Face à une langue piégée, le traducteur doit avancer, si j'ose dire, sur le bout des doigts: il ne faut ni jouer le jeu des bourreaux ni atténuer la violence de leur langage. L'une des solutions consiste à traduire celui-ci en tenant compte de toutes ses particularités: la violence qui lui donne son efficacité politique, certes, mais également la lourdeur, les incorrections, la niaiserie et l'exaltation propres à la quasi-totalité de leurs discours. C'est ce que j'ai pu faire aussi bien sur le Journal de Goebbels, document historique passionnant autant qu'illustration des aberrations de la pensée du ministre de l'Éducation et de la Propagande, que dans la traduction de Mein Kampf qui paraîtra en $2020^{11}$ sous la direction de Florent Brayard - et qui restituera le chaos mental et linguistique que représentait ce texte.

Parce que les derniers témoins de la Shoah sont en train de disparaître, parce que les dimensions humaines et éthiques de ce crime sont sans précédent dans l'histoire, il reste indispensable de travailler sur cette période, et donc de traduire études et sources. Encore faut-il se donner toutes les armes pour que ces traductions conservent les caractéristiques des originaux sans entrer dans le jeu pervers auquel leurs auteurs se sont livrés avec le langage et qui a transformé leur prose en champ

10. Texte intégral en français: http://phdn.org/histgen/documents/nazisdoc.html himmler-19431006

11. Aux éditions Fayard, édition critique et largement annotée par une nombreuse équipe de spécialistes. 
de mines. Elle nous menace toujours aujourd'hui, et le traducteur est l'un de ceux qui peuvent et doivent tenter de la neutraliser.

\section{olivier.mannoni@wanadoo.fr}

Olivier Mannoni a publié à ce jour plus de deux cents traductions, dont une quarantaine porte sur le nazisme. Directeur pédagogique de I'École de traduction littéraire (ETL, un partenariat CNL/Asfored) dont il a conçu les principes en 2012, il a reçu en 2018 le prix Eugen Helmlé pour l'ensemble de son œuvre. 